\title{
A relação estresse e hardiness em acadêmicos de enfermagem de uma instituição de ensino superior
}

The relationship stress and hardiness in academics of nursing in a higher education institution

La relación estrés y hardela en académicos de enfermería de una institución de enseñanza superior

Ana Patrícia Fonseca Coelho GALVÃO ${ }^{(1)}$ Abel dos Santos SOUZA NETO ${ }^{(2)}$

Recebido: 23 abr 2018 Revisado: 2 jun 2018 Aceito: 9 nov 2018

Autor de

correspondência: Ana Patrícia Fonseca Coelho Galvão apfcoelho@gmail.com

Conflito de interesses: Os autores declaram não haver nenhum interesse profissional ou pessoal que possa gerar conflito de interesses em relação a este manuscrito.
(1) Faculdade Pitágoras, São Luís, MA, Brasil.

(2) Universidade Ceuma, São Luís, MA, Brasil.

\begin{abstract}
Resumo
O estresse, uma reação natural do organismo que ocorre quando vivenciamos situações de perigo ou ameaça. Para minimizar o estresse, sua influência na saúde e suas repercussões negativas na vida social são utilizadas algumas estratégias. Dentre elas, Hardiness, ou Personalidade Resistente que são pessoas que podem ter características refratárias ao estresse. Foi realizado um estudo descritivo exploratório com abordagem quantitativa onde foi aplicado um questionário para a caracterização da amostra e a Escala de Hardiness em 60 acadêmicos de enfermagem. Os dados coletados foram analisados nos programas EPI-INFO versão 3.5.1 e STATA ${ }^{\circledR}$, versão 10.0, para gerenciamento de dados e análises dos mesmos. As maiorias dos entrevistados do curso de Enfermagem $55 \%$ estão entre os $7^{\circ}$ e $10^{\circ}$ períodos, a maior parte da amostra se concentra no período noturno, no entanto $55 \%$ dos acadêmicos possuem emprego no horário inverso ao que estudam $35,3 \%$, possuem reprovações ou dependência $55 \%$ da amostra total deste estudo. Na relação estresse e hardiness, 9 acadêmicos apresentaram características hardy 15\%, ou seja, apresentam característica resistente, 51 dos entrevistados não apresentam características hardy $85 \%$, o que nos leva a crer que são indivíduos estressados. Contudo, ser hardy não significa eliminar os estressores, mas modificar a percepção do estresse, mobilizando estratégias para um melhor enfrentamento, tendo resistência por meio de uma capacidade maior de tolerância às situações e eventos, por ser hardiness um constructo da personalidade, é passível de ser apreendida. A pesquisa obedeceu aos aspectos éticos e legais de pesquisa em seres humanos.
\end{abstract}

Descritores: Personalidade resistente; Estresse; Exaustão Emocional. 


\begin{abstract}
Stress, a natural reaction of the body that occurs when we experience situations of danger or threat. To minimize stress, its influence on health and its negative repercussions on social life are used some strategies. Among them, Hardiness, or Resilient Personality are people who may have stressrelieving characteristics. An exploratory descriptive study with a quantitative approach was carried out in which a questionnaire was applied to the characterization of the sample and the Hardiness Scale in 60 nursing students. The collected data were analyzed in the programs EPI-INFO version 3.5.1 and STATA $®$, version 10.0, for data management and analysis of the same. Most of the interviewees of the Nursing course $55 \%$ are in the $7^{\text {th }}$ and $10^{\text {th }}$ periods, most of the sample is concentrated in the night time, however $55 \%$ of the students have jobs in the opposite time frame to that studied $35.3 \%$, have disapprovals or dependence $55 \%$ of the total sample of this study. In the relation stress and hardiness, 9 academics presented hardy characteristics $15 \%$, that is, they present resistant characteristics, 51 of the interviewed do not present hardy characteristics $85 \%$, which leads us to believe that they are stressed individuals. However, being hardy does not mean eliminating stressors, but modifying the perception of stress, mobilizing strategies for better coping, having resistance through a greater capacity of tolerance to situations and events, being hardiness a personality construct, is capable of be seized. The research obeyed the ethical and legal aspects of human research.
\end{abstract}

Keywords: Resistant Personality; Stress; Emotional Exhaustion.

\title{
Resumen
}

El estrés, una reacción natural del organismo que ocurre cuando vivimos situaciones de peligro o amenaza. Para minimizar el estrés, su influencia en la salud y sus repercusiones negativas en la vida social se utilizan algunas estrategias. Entre ellas, Hardiness, o Personalidad Resistente que son personas que pueden tener características refractarias al estrés. Se realizó un estudio descriptivo exploratorio con abordaje cuantitativo donde se aplicó un cuestionario para la caracterización de la muestra y la Escala de Hardiness en 60 académicos de enfermería. Los datos recogidos fueron analizados en los programas EPI-INFO versión 3.5.1 y STATA ${ }$, versión 10.0, para gestión de datos y análisis de los mismos. Las mayorías de los entrevistados del curso de Enfermería $55 \%$ están entre los $7^{\circ}$ y $10^{\circ}$ períodos, la mayor parte de la muestra se concentra en el período nocturno, sin embargo, el 55\% de los académicos poseen empleo en el horario inverso al que estudian el 35,3\%, poseen reprobaciones o dependencia del $55 \%$ de la muestra total de este estudio. En la relación estrés y hardiness, 9 académicos presentaron características hardy 15\%, o sea, presentan característica resistente, 51 de los entrevistados no presentan características hardy $85 \%$, lo que nos lleva a creer que son individuos estresados. Sin embargo, ser hardy no significa eliminar los estresores, sino modificar la percepción del estrés, movilizando estrategias para un mejor enfrentamiento, teniendo resistencia por medio de una capacidad mayor de tolerancia a las situaciones y eventos, por ser hardiness un constructo de la personalidad, es pasible ser aprehendida. La investigación obedeció a los aspectos éticos y legales de investigación en seres humanos.

Palabras-claves: Personalidad resistente; El estrés; Extracción Emocional.

\section{Introdução}

Atualmente, a crescente e exacerbada utilização do termo estresse, tem sido utilizada de forma trivial e às vezes, instintiva. Diversos fatores biopsicossociais são 
desencadeadores de situações estressantes, fatores esses encontrados nos mais diversos ambientes como, por exemplo, na vida pessoal, social, no ambiente de trabalho, e não menos diferente, durante a trajetória acadêmica, pois o estudante de enfermagem, ao ingressar na vida acadêmica, passa por diversas situações de crise, vivenciando diversos sentimentos. Destacando-se a formação de um novo ciclo de amigos, adaptação aos novos horários, problemas financeiros, preocupações com seu futuro mercado de trabalho, afastamento do seu ambiente familiar, as cobranças, o encontro com a dor, o sofrimento, a doença e a morte de pessoas são pontos que favorecem o desequilíbrio emocional. Todos esses fatores podem ser entendidos como estressores, desencadeando o stress e fazendo com que o sujeito não consiga se adaptar a novas situações. ${ }^{1}$

Para minimizar o estresse, suas influências na saúde e suas repercussões negativas na vida social são utilizadas algumas estratégias. Dentre elas destaca-se, Hardiness ou Teoria da Personalidade Resistente, a qual tem seu alicerce em três componentes: compromisso, controle e desafio. ${ }^{2}$

Hardiness (Personalidade Resistente) são pessoas que podem ter características refratárias ao estresse, características essas que servem como contrapartida aos estressores. Nesse sentido, hardiness pode ser definido como a capacidade das pessoas em enfrentar os estressores como um desafio o que permite o desenvolvimento de experiências, possibilitando o conhecimento. ${ }^{3}$

Hardiness pode ser definido como coragem existencial, ou seja, coragem de seguir em frente, de encarar a vida e além de tudo, promove motivação para enfrentar tarefas difíceis, além de ter efeitos diretos e indiretos na saúde e bem-estar, pois os indivíduos com essas características (indivíduos hardy) têm maneiras diferentes de enfrentar o estresse, se comportam de forma mais saudável e robusta, realizando uma forma adaptável, tanto fisiológica quanto psicológica de bem estar. ${ }^{4}$

O tema começa a ser foco de pesquisas, com o aumento do debate crítico, referente à sua utilização nas organizações e na vida individual. Contudo, pouca alteração com 
relação ao conceito, aplicação e medida. Ainda assim, alguns pesquisadores estão trazendo a discussão os fatores sociais que afetam a saúde o bem-estar dos indivíduos. ${ }^{5}$

A avaliação dos níveis de hardiness em acadêmicos de enfermagem podem fornecer informações simples, mas importantes, para os estudantes, enfermeiros e s instituições sobre como enfrentar o estresse. Pelo fato de poder ser apreendido, há a possibilidade de hardiness ser aumentado por meio de atividades de aprendizagem, impactando na qualidade de vida e de saúde do profissional, decorrendo em uma melhor qualidade do trabalho desenvolvido, por conseguinte. ${ }^{5}$

Com base no pressuposto, a diversidade de questionamentos a respeito do tema abordado como, por exemplo, o porquê de analisar os níveis de hardiness e sua relação com o estresse e que vantagens o acadêmico ganharia com essas informações. Desta forma, a justificativa deste trabalho encontra-se no fato da análise dos níveis de hardiness durante a trajetória acadêmica para que possa ser um agente de mudança e gerar subsídios para que os acadêmicos de enfermagem possam ter estratégias de enfrentamento das situações de estresse vivenciadas durante esse período de suas vidas, tornando o acadêmico mais satisfeito no exercício de suas atividades e diminuindo a influência do estresse na sua vida pessoal.

Em prol do acima citado este estudo teve como principal objetivo avaliar a relação estresse e hardiness em acadêmicos de enfermagem de uma instituição de ensino superior em São Luís, Maranhão.

\section{Metodologia}

O desenvolvimento do tema proposto foi realizado por um estudo descritivo, exploratório com abordagem quantitativa, desenvolvido em uma Instituição de Ensino Superior do município de São Luís-MA. Tal instituição, uma universidade particular, e conta com um grande número de acadêmicos, matriculados no curso de enfermagem nos 
turnos, matutino e noturno. A população foi constituída de 487 alunos regularmente matriculados no curso de enfermagem desta instituição, e que aceitaram participar do estudo por meio da assinatura do Termo de Consentimento Livre e Esclarecido -TCE. Foram incluídos neste estudo acadêmicos do curso de Enfermagem de ambos os sexos, que estavam no exercício de suas atividades e excluídos acadêmicos afastados por motivo de doença e licença maternidade.

Para a coleta de dados se utilizou um questionário autoaplicado para descrição do perfil socioeconômico e dos fatores influenciam no estresse. Garantindo anonimato, uma vez que as análises pretendidas não são a nível individual, mas análises estatísticas de natureza geral. Utilizou-se a Escala de Hardiness, uma versão adaptada, da Escala de Bartone e colaboradores, traduzida e validada da língua inglesa para a língua portuguesa, que tem por objetivo verificar a quantidade de personalidade resistente ao estresse. É composta por 30 itens subdivididos nos domínios: 10 itens para Compromisso, 10 para Controle e 10 para Desafio. O projeto foi submetido no Comitê de Ética em Pesquisa e após sua aprovação através do Parecer Consubstanciado $\mathrm{n}^{\circ} 1.069 .932$, se iniciou a coleta de dados.

Os dados coletados foram processados e analisados a partir dos programas EPIINFO 2008 versão 3.5.1 (CDC, Atlanta, EUA) e STATA ${ }^{\circledR}$, versão 10.0, para gerenciamento de dados e análises dos mesmos.

\section{Resultados}

Os resultados foram processados e apresentados de forma percentual para melhor compreensão dos mesmos.

Podemos observar na Tabela 1 que $70 \%$ da amostra total deste estudo correspondem ao gênero feminino e estão com média de idade entre 16 e 21 anos, destes $56,6 \%$ estão solteiros. Quando questionados sobre o período em que se encontram no 
curso de Enfermagem $55 \%$ estão entre os $7^{\circ}$ e $10^{\circ}$ períodos, a maior parte da amostra se concentra no período noturno, no entanto $55 \%$ dos acadêmicos possuem emprego no horário inverso ao que estudam e $35,3 \%$ dos alunos residem com os pais enquanto $68,4 \%$ não possuem filhos, e 55\% da amostra total possuem reprovações ou disciplinas em dependência.

Tabela 1. Distribuição percentual do perfil socioeconômico dos acadêmicos do curso de Enfermagem entrevistados.

\begin{tabular}{|c|c|c|}
\hline Variável & $\mathbf{N}$ & $\%$ \\
\hline \multicolumn{3}{|l|}{ Gênero } \\
\hline Masculino & 18 & 30 \\
\hline Feminino & 42 & 70 \\
\hline \multicolumn{3}{|l|}{ Faixa Etária } \\
\hline $16-21$ & 23 & 38,4 \\
\hline $22-29$ & 22 & 36,6 \\
\hline 30 ou mais & 15 & 25 \\
\hline \multicolumn{3}{|l|}{ Estado Civil } \\
\hline Casado & 12 & 20 \\
\hline Solteiro & 34 & 56,6 \\
\hline União estável & 14 & 23,4 \\
\hline \multicolumn{3}{|l|}{ Período } \\
\hline 1 - $3^{\circ}$ Período & 12 & 20 \\
\hline $4-6^{\circ}$ Período & 15 & 25 \\
\hline $7-10^{\circ}$ Período & 33 & 55 \\
\hline \multicolumn{3}{|l|}{ Turno } \\
\hline Matutino & 28 & 46,6 \\
\hline Noturno & 32 & 53,4 \\
\hline \multicolumn{3}{|l|}{ Reprovação ou } \\
\hline \multicolumn{3}{|l|}{ Dependência } \\
\hline Sim & 33 & 55 \\
\hline Não & 27 & 45 \\
\hline \multicolumn{3}{|l|}{ Reside } \\
\hline Sozinho & 14 & 23,3 \\
\hline Amigos & 7 & 11,6 \\
\hline Com os pais & 21 & 35,3 \\
\hline Familiares & 13 & 21,3 \\
\hline Outros & 5 & 8,2 \\
\hline \multicolumn{3}{|l|}{ Tem filhos } \\
\hline Sim & 19 & 31,6 \\
\hline Não & 41 & 68,4 \\
\hline
\end{tabular}




\begin{tabular}{ccc}
\hline Trabalha & & \\
Sim & 33 & 55 \\
Não & 27 & 45 \\
\hline Total & 60 & 100 \\
\hline
\end{tabular}

Fonte: Elaborado pelos autores (2017).

Podemos observar, no Gráfico 1, que foi calculada a média das variáveis da Escala de Hardiness, onde a variável Compromisso, correspondeu a 17,15. O variável Controle apresentou uma média correspondente a 18\%, a variável Desafio apresentou valor médio de 14,65 .

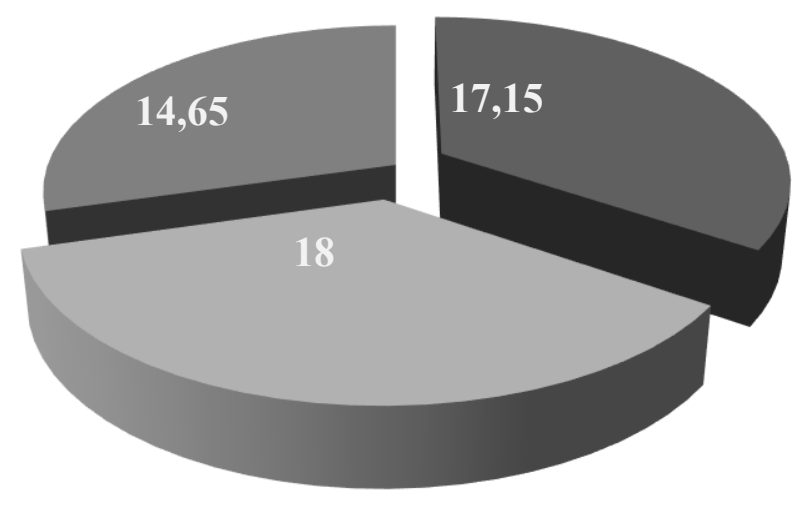

- Compromisso

Controle

Desafio

Gráfico 1. Distribuição da média de valores da análise da Escala de Hardiness em acadêmicos do curso de Enfermagem.

Fonte: Elaborado pelos autores (2017).

A Tabela 2 mostra a distribuição numérica e percentual de cada variável da escala de Hardiness, onde no variável Compromisso foram observados nove acadêmicos, o que corresponde a $15 \%$ da amostra que pontuaram valor igual ou acima de 20 , sendo este 0 valor de referência para identificar se o acadêmico possui pelo menos uma variável compatível, entretanto para ser classificado como um indivíduo hardy ele deve apresentar pelo menos duas variáveis com valores iguais ou maiores que 20. Na variável Controle foram observados 15 acadêmicos, correspondendo a $25 \%$ da amostra, com característica hardy. Para variável Desafio, foram identificados apenas seis indivíduos, o que corresponde a $10 \%$ da amostra. 
Tabela 2. Distribuição percentual de cada variável da escala de Hardiness de acordo com o valor de referência apresentado na metodologia.

\begin{tabular}{lccccc}
\multirow{2}{*}{ Variável } & \multicolumn{2}{c}{ Sim } & \multicolumn{2}{c}{ Não } & \multicolumn{2}{c}{ Total } \\
\cline { 2 - 6 } & $\mathbf{n}$ & \% & $\mathbf{n}$ & $\%$ & $\%$ \\
Compromisso & 9 & 15 & 51 & 85 & 100 \\
Controle & 15 & 25 & 45 & 75 & 100 \\
Desafio & 6 & 10 & 54 & 90 & 100 \\
\hline
\end{tabular}

Fonte: Elaborado pelos autores (2017).

O Gráfico 2 mostra a relação estresse e hardiness entre os acadêmicos do curso de enfermagem entrevistados, onde, $15 \%$ da amostra, o que equivale a 9 acadêmicos, apresentam características hardy, ou seja, apresentam característica resistente, e $85 \%$ dos acadêmicos, correspondendo a 51 dos entrevistados, não apresentam características hardy o que se pode supor que são indivíduos estressados.

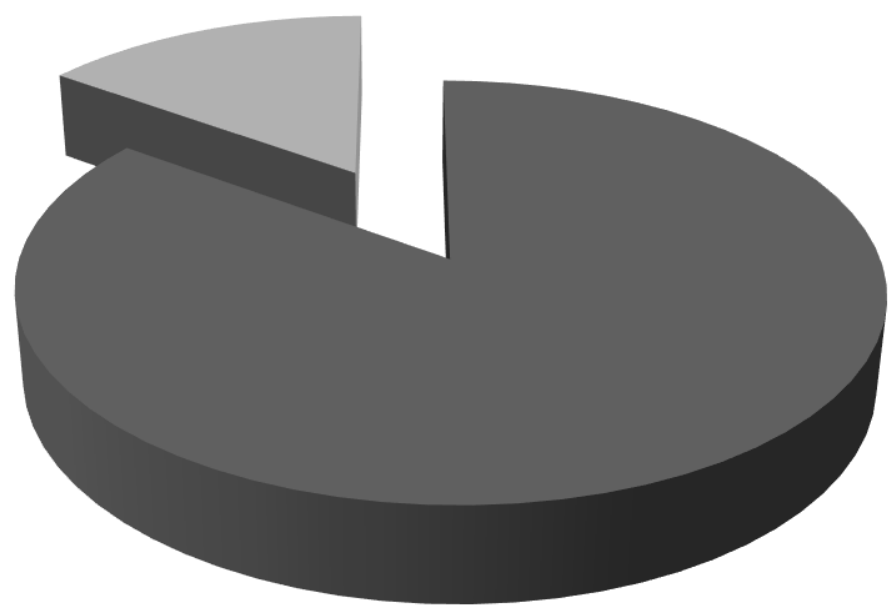

Estresse

Hardiness

Gráfico 2. Distribuição percentual da análise da relação estresse e hardiness em acadêmicos do curso de Enfermagem entrevistados.

Fonte: Elaborado pelos autores (2017). 


\section{Discussão}

Pode-se correlacionar o gênero feminino em evidência no presente estudo, com os resultados achados na pesquisa de Donoso, ${ }^{6}$ no qual considera a Enfermagem como uma profissão com predominância do sexo feminino, pois há uma relação histórica entre esse predomínio e o cuidado - atividade referencial da profissão. Também se observou que a maioria dos acadêmicos está em uma média de idade entre 16 e 21 anos e são solteiros, dados que corroboram com o estudo de Wetterich e Melo, ${ }^{7}$ uma vez que é o período em que eles saem do ensino médio para ingressar na vida acadêmica e paralelamente a isso não se interessam em casar e não terem filhos, variável que ficou nítida neste estudo.

A maioria dos entrevistados se encontra entre $\circ 7^{\circ}$ e o $10^{\circ}$ período, estudam no turno noturno e trabalham e se compara com o estudo de Alves ${ }^{8}$ pois o perfil dos estudantes de enfermagem é o de ser "trabalhador estudante", enfrentando, na grande maioria, dupla ou tripla jornada de trabalho. Em consequência das más condições de vida, apresentadas frequentemente por eles, chegam atrasados ou dormem durante as aulas, podendo interferir em sua qualidade de vida e consequentemente no processo de ensinoaprendizagem o que acarreta no maior número de reprovação ou dependência, variável que também se destacou neste estudo.

Podemos correlacionar este estudo com o de Batista ${ }^{5}$ onde a média de hardiness em suas subescalas, sendo possível constatar valores semelhantes para as subescalas compromisso e controle e valor um pouco inferior para a subescala Desafio.

As três dimensões do hardiness (controle, compromisso e desafio) apresentam-se como uma forma de proteção ou resistência frente a agentes estressores. No estudo de Andrade $^{9}$ nas dimensões Compromisso e Controle, as médias dos valores foram classificadas como significativas para hardiness e a variável Desafio com média inferior, apresentou-se como baixa para hardiness. Dados que também corroboraram com o presente estudo. O autor afirma ainda, que o estilo hardy de ser, é um modo generalizado de funcionamento que inclui de forma equilibrada um forte senso de comprometimento, 
uma crença de que é possível controlar ou influenciar fatores externos, explorando uma abordagem de vida (desafio) e uma orientação para o futuro.

Os dados da Tabela 2 são semelhantes ao estudo de Kirchhof, ${ }^{10}$ onde os indivíduos com alto compromisso se sentem comprometidos com o trabalho ou estudo, a ponto de conseguirem agir de maneira positiva frente aos estressores. Esse domínio se caracteriza pela capacidade que as pessoas têm em receber propostas e tomar decisões, além de apoiar o equilíbrio interno e garantir a competência para lidar com situações consideradas estressoras.

Batista e Bianchi ${ }^{2}$ ressaltam que o hardiness Desafio transforma as pessoas estimuladas em seu ambiente proporcionando capacidade de resposta ao inesperado. Essa antecipação exige que as pessoas explorem ou identifiquem em seu ambiente os recursos que podem ajudá-las a lidar com o estresse.

$\mathrm{O}$ estudo de Martins ${ }^{11}$ descreve a relação negativa entre hardiness e estresse em acadêmicos, identificando que os alunos com uma personalidade mais resistente não eram tão afetados pelos efeitos nocivos do estresse acadêmico, sugerindo, ainda, que não é a situação estressante em si que desencadeia estresse, mas sim a forma como o sujeito reage e a percepciona.

\section{Conclusão}

Na pesquisa pode-se concluir que os níveis de hardiness $15 \%$ são bastante baixos quando correlacionados com o estresse $85 \%$, repercutindo de maneira negativa na vida do acadêmico, que assume uma postura de passividade e acomodação.

Verificou-se também, que dos indivíduos que apresentam personalidade hardy 15\%, apresentaram predisposição ao compromisso, com média identificada igual a 17,15. Para a variável controle, a média identificada foi igual a 18, indicando acadêmicos empenhados em suas atividades, envolvidos com as pessoas e com o que acontece ao seu redor. Dessa 
forma se esforçam para influenciar seus resultados de forma positiva, mesmo que isso possa parecer difícil em determinadas circunstâncias. Entretanto, a média encontrada para a variável Desafio foi 14,65, apresentando deficiência neste campo. E escores baixos nesta variável indicam que os indivíduos são absorvidos pelo desejo de comodidade e segurança.

Contudo, ser hardy não significa eliminar os estressores, mas modificar a percepção do estresse, mobilizando estratégias para um melhor enfrentamento, desta forma, tendo resistência por meio de uma capacidade maior de tolerância às situações e eventos, construindo uma personalidade, que é passível de ser apreendida. Acreditamos que os resultados representam avanços e poderão auxiliar a coordenação do curso de enfermagem com a proposição de ações de intervenção na estrutura curricular e no cotidiano acadêmico, a fim de torná-lo mais produtivo e menos desgastante.

\section{Referências}

1. Monteiro CFS, Freitas JFM, Ribeiro AAP. Estresse no cotidiano acadêmico: o olhar dos alunos de enfermagem da Universidade Federal do Piauí. Esc Anna Nery R Enferm. 2007;11(1):66-72.

2. Batista KM, Bianchi ERF. A relação estresse, hardness e turno de trabalho em enfermeiros de um hospital de ensino. Enferm Glob. 2013;12(29):281-7.

3. Silva RM, Goulart CT, Bolzan MEO, Serrano PM, Lopes LFD, Guido LA. Estresse e Hardness em residentes médicos. Rev Enferm UFPE on line. 2013;7(9):5406-13. http://dx.doi.org/10.5205/reuol.3529-29105-1-SM.0709201306.

4. Serrano PM. Adaptação cultural da hardness scale (HS) [tese]. São Paulo: Universidade de São Paulo Escola de Enfermagem; 2009. http://dx.doi.org/10.11606/D.7.2009.tde11012010-121444.

5. Batista KM. Estresse e hardness entre enfermeiros hospitalares [tese]. São Paulo: Universidade de São Paulo; 2011. http://dx.doi.org/10.11606/T.7.2011.tde-31052011120626.

6. Donoso VTM. O gênero e suas possíveis repercussões na gerência de enfermagem. REME. 2000;4(1-2):67-9. 
7. Wetterich $C N$, Melo MRAC. Perfil sociodemográfico do aluno do curso de graduação em enfermagem. Rev Latino-Am Enferm. 2007;15(3):404-10. http://dx.doi.org/10.1590/S0104-11692007000300007.

8. Alves FE. Qualidade de vida de estudantes de enfermagem de uma universidade privada. R Bras Qual Vida. 2010;2(1):23-30. http://dx.doi.org/10.3895/S217508582010000100003.

9. Andrade JS. Estresse ocupacional, hardiness e qualidade de vida profissional de policiais militares de uma cidade do interior de Mato Grosso do Sul [tese]. Campo Grade: Universidade Católica Dom Bosco; 2013.

10. Kirchhof RS. Estresse, coping, síndrome de Burnout, sintomas depressivos e Hardiness em docentes de Enfermagem [dissertação]. Santa Maria: Universidade Federal de Santa Maria; 2013

11. Martins RP. Stress percebido, hardness e satisfação com a vida nos alunos da Academia Militar: relação entre variáveis [dissertação]. Lisboa: Universidade de Lisboa; 2013.

\section{Minicurrículo}

Ana Patrícia Fonseca Coelho Galvão | ORCiD: 0000-0003-3376-5678

Professora na Faculdade Pitágoras. Mestre em Saúde em Saúde e Ambiente pela Universidade Federal do Maranhão - UFMA. Especialista em Saúde Mental e Saúde da Família pela Universidade Estácio de Sá, RJ.

Abel dos Santos Souza Neto | ORCiD: 0000-0003-1063-6048

Enfermeiro, Especialista em Unidade de Terapia Intensiva pela Universidade Ceuma. 